\title{
Bone marrow dosimetry in peptide receptor radionuclide therapy with $\left[{ }^{177} \mathrm{Lu}-\mathrm{DOTA}{ }^{0}\right.$, Tyr $\left.^{3}\right]$ octreotate
}

\author{
Flavio Forrer • Eric P. Krenning • Peter P. Kooij • Bert F. Bernard • \\ Mark Konijnenberg • Willem H. Bakker • Jaap J. M. Teunissen • Marion de Jong • \\ Kirsten van Lom • Wouter W. de Herder • Dik J. Kwekkeboom
}

Received: 18 July 2008 / Accepted: 30 December 2008 / Published online: 27 February 2009

(C) The Author(s) 2009. This article is published with open access at Springerlink.com

\begin{abstract}
Purpose Adequate dosimetry is mandatory for effective and safe peptide receptor radionuclide therapy (PRRT). Besides the kidneys, the bone marrow is a potentially doselimiting organ. The radiation dose to the bone marrow is usually calculated according to the MIRD scheme, where the accumulated activity in the bone marrow is calculated from the accumulated radioactivity of the radiopharmaceutical in the blood. This may underestimate the absorbed dose since stem cells express somatostatin receptors. We verified the blood-based method by comparing the activity in the blood with the radioactivity in bone marrow aspirates. Also, we evaluated the absorbed cross-dose from the source organs (liver, spleen, kidneys and blood), tumours and the so-called "remainder of the body" to the bone marrow.

Methods Bone marrow aspirates were drawn in 15 patients after treatment with $\left[{ }^{177} \mathrm{Lu}-\mathrm{DOTA}^{0}, \mathrm{Tyr}^{3}\right]$ octreotate. Radioactivity in the bone marrow was compared with radioactivity in
\end{abstract}

F. Forrer $(\bowtie) \cdot$ E. P. Krenning $\cdot$ P. P. Kooij · B. F. Bernard

W. H. Bakker · J. J. M. Teunissen $\cdot$ M. de Jong $\cdot$

D. J. Kwekkeboom

Department of Nuclear Medicine, Erasmus MC Rotterdam,

Dr. Molewaterplein 40,

NL-3015 GD Rotterdam, The Netherlands

e-mail: fforrer@uhbs.ch

M. Konijnenberg

Research and Development, Mallinckrodt Medical BV,

Covidien,

Petten, The Netherlands

K. van Lom

Department of Haematology, Erasmus MC Rotterdam,

Rotterdam, The Netherlands

W. W. de Herder

Department of Internal Medicine, Erasmus MC Rotterdam,

Rotterdam, The Netherlands the blood drawn simultaneously. The nucleated cell fraction was isolated from the bone marrow aspirate and radioactivity was measured. The absorbed dose to the bone marrow was calculated. The results were correlated to the change in platelet counts 6 weeks after treatment.

Results A strong linear correlation and high agreement between the measured radioactivities in the bone marrow aspirates and in the blood was found $(r=0.914, p<0.001)$. No correlation between the calculated absorbed dose in the bone marrow and the change in platelets was found. There was a considerable contribution from other organs and the remainder of the body to the bone marrow absorbed dose.

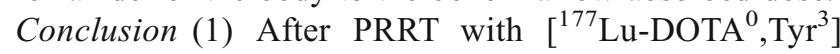
octreotate, the radioactivity concentration in the bone marrow is identical to that in the blood; (2) There is no significant binding of the radiopharmaceutical to bone marrow precursor stem cells; (3) The contribution of the cross dose from source organs and tumours to the bone marrow dose is significant; and (4) There is considerable variation in bone marrow absorbed dose between patients. These findings imply that for individual dose optimization, individual calculation of the bone marrow absorbed dose is necessary.

Keywords Dosimetry $\cdot$ Bone marrow $\cdot\left[{ }^{177}\right.$ Lu-DOTA $^{0}$, Tyr $\left.^{3}\right]$ octreotate $\cdot$ Therapy $\cdot$ Somatostatin receptor

\section{Introduction}

Peptide receptor radionuclide therapy (PRRT) with radiolabelled somatostatin analogues, such as $\left[{ }^{90} \mathrm{Y}^{-D O T A}{ }^{0}, \mathrm{Tyr}^{3}\right]$ octreotide $\left({ }^{90}\right.$ Y-DOTATOC) or $\left[{ }^{177}\right.$ Lu-DOTA $\left.^{0}, \mathrm{Tyr}^{3}\right]$ octreotate, has been shown to be an effective treatment in patients with metastatic neuroendocrine tumours [1-6]. The dose-limiting 
organs with this treatment are usually either the kidneys or the bone marrow [7-9]. In order to avoid toxicity due to absorbed radiation dose, dosimetry can be performed. While kidney dosimetry has been investigated extensively [10-12], less attention has been paid to bone marrow dosimetry.

In order to achieve a maximum antitumour effect, patients should be treated with the highest justifiable dose of the radiopharmaceutical that does not cause serious toxicity. Often the kidneys are the dose-limiting organ. However, recently it has been shown that the bone marrow has to be regarded as the dose-limiting organ in approximately $70 \%$ of patients treated with $\left[{ }^{177} \mathrm{Lu}-\mathrm{DOTA}^{0}, \mathrm{Tyr}^{3}\right]$ octreotate [13]. Many studies with radiolabelled somatostatin analogues have shown that the myelotoxicity is generally mild and transient $[1-3,6]$. However, in a phase 1 study with $\left[{ }^{111}\right.$ In-DTPA $\left.{ }^{0}\right]$ octreotide 3 out of 50 patients developed a myelodysplastic syndrome (MDS) which was probably related to the therapy [14]. Calculations from these data resulted in an estimated radiation absorbed dose for the bone marrow of approximately 3 Gy. In another study with $\left[{ }^{177} \mathrm{Lu}-\mathrm{DOTA}{ }^{0}, \mathrm{Tyr}^{3}\right]$ octreotate, MDS was observed in a patient who had had chemotherapy with alkylating agents 2 years before study entry [15]. In the latest update of our own records of 504 patients treated with $\left[{ }^{177} \mathrm{Lu}-\mathrm{DOTA}{ }^{0}, \mathrm{Tyr}^{3}\right]$ octreotate, four patients (including the patient mentioned above) developed MDS [16].

To avoid hypoplasia, a maximum absorbed dose of 2 Gy to the bone marrow is generally accepted [2, 17]. With a dose of $2 \mathrm{~Gy}$, the probability for developing leukaemia is approximately $2 \%$ [18]. In radioiodine therapy of metastatic thyroid cancer a threshold of 2 Gy to the blood as surrogate for bone marrow has been maintained since the pioneering work of Benua et al. in 1962 [19]. More recent work has set this limit to as high as 3 Gy by using more patient-specific dosimetry techniques [20]. Nevertheless, even if this limit is not exceeded the risk of the patient developing MDS cannot be excluded completely, but an accurate estimation of the absorbed dose to the bone marrow will help find an adequate dosage.

The absorbed dose to the bone marrow during radionuclide therapies may be estimated with the aid of the MIRD scheme [21-23]. Contributions to the red marrow dose $\left(D_{r m}\right)$ arise from the accumulated activity in the marrow tissue itself $\left(\widetilde{A}_{r m}\right)$ (self-dose), in source organs (including tumours and blood) $\widetilde{A}_{h}$ and in the remainder of the body $\widetilde{A}_{r b}$ :

$D_{r m}=\widetilde{A}_{r m} S_{r m \leftarrow r m}+\sum \widetilde{A}_{h} S_{r m \leftarrow h}+\widetilde{A}_{r b} S_{r m \leftarrow r b}$

with $S_{r m \leftarrow r m}$ the self-dose S-factor, $S_{r m \leftarrow h}$ the cross-dose S-factor of the source organs and $S_{r m \leftarrow r b}$ the cross-dose Sfactor of the remainder of the body. The accumulated activity of the radiopharmaceutical in the bone marrow is calculated from the accumulated activity in the blood (Eq. 2)

$\widetilde{A}_{r m}=[\widetilde{A}]_{b l} \times R M B L R \times\left\{\begin{array}{c}1.3 / 57(\text { female }) \\ 1.1 / 74(\text { male })\end{array}\right\} \times M_{p a t}$

where $[\widetilde{A}]_{b l}$ is the accumulated activity concentration in the blood, $1.3 / 57$ and $1.1 / 74$ are the ratios between the red marrow mass and the total body mass for the adult reference woman and the adult reference man, respectively, and $M_{p a t}$ is the mass of the patient in kilograms. Taking into consideration the red marrow to blood concentration ratio (RMBLR), a correction factor can be added depending on the vector used for treatment. This method has been validated by bone marrow aspiration for antibodies but not for radiopeptides $[24,25]$.

For radiolabelled somatostatin analogues, the calculation usually deals with the assumption of absence of specific uptake of the radiolabel into the bone marrow. Since certain haematological cells (lymphocytes, monocytes) and haematopoietic progenitor cells express somatostatin receptors, mainly subtype 2 [26-28], this assumption may result in an underestimation of the absorbed radiation dose to the bone marrow. It is not known whether in patients a significantly higher radioactivity in the bone marrow, compared to the blood, may be present.

Relevant results for bone marrow dosimetry were obtained using ${ }^{86} \mathrm{Y}$ as a surrogate for ${ }^{90} \mathrm{Y}$ to calculate the accumulated dose in the red marrow by PET before treatment with ${ }^{90}$ Y-DOTATOC. A region of interest (ROI) was drawn around a segment of the thoracic spine and rescaled for the whole red marrow mass using the standard fraction of active red marrow present in the thoracic spine [29]. Recently a comparable calculation showing relevant results as well was presented for iodinated antibodies. Instead of a PET scan patients underwent a SPECT/CT scan during therapy [30]. Acquiring a CT scan and using an integrated SPECT/CT camera allows the ROIs to be placed anatomically more accurately than using SPECT alone. This is especially important in radiopharmaceuticals, e.g. most radiopeptides, which do not show sufficiently high bone marrow uptake to be indisputably identified on the scans. In addition the $\mathrm{CT}$ scan provides an attenuation map that can be used to apply an attenuation correction. With three SPECT scans at different time-points after therapy, we investigated the feasibility of this calculation in a therapeutic setting in this study.

In order to verify whether the assumption of no specific uptake in the bone marrow can be applied with radiolabelled somatostatin analogues, we compared the radioactivity in bone marrow aspirates with the radioactivity in blood samples drawn simultaneously. In addition we 
determined the difference between the radioactivity in the nucleated cell fraction of the bone marrow, including the stem cells, and that in the blood to determine whether there was any difference that could be attributed to the specific binding of the radiopeptide to somatostatin receptorpositive cells in the bone marrow. Finally, the absorbed doses to the bone marrow calculated according to the MIRD scheme and from the SPECT scans were correlated with the change in the platelet counts 6 weeks after the treatment.

\section{Materials and methods}

\section{Patients}

Included in the study were 15 patients with somatostatin receptor-positive neuroendocrine tumours. All patients were admitted to our clinic for PRRT with $\left[{ }^{177} \mathrm{Lu}-\mathrm{DOTA}{ }^{0}, \mathrm{Tyr}^{3}\right]$ octreotate and fulfilled the inclusion criteria as previously described [6]. None of the patients had known bone metastases. All patients gave written informed consent to participate in the study, which was approved by the medical ethics committee of the hospital.

Comparison of the radioactivity in the bone marrow and nucleated cell fraction with the radioactivity in the blood

In addition to the examinations, the treatment, and the scans that are performed routinely during and after the first treatment cycle with $\left[{ }^{177} \mathrm{Lu}-\mathrm{DOTA}{ }^{0}, \mathrm{Tyr}^{3}\right]$ octreotate, patients underwent bone marrow aspiration from the iliac crest 4 days (seven patients), 7 days (seven patients) or 8 days (one patient) after the treatment. A rough differential count of cells was performed on the bone marrow samples to prove the presence of a sufficient number of bone marrow cells and the samples were analysed for haematological abnormalities. The volume of aspirate was recorded and the radioactivity was measured in a gamma counter (Perkin Elmer, Groningen, The Netherlands). One patient (aspiration 4 days after treatment) was excluded because no bone marrow could be aspirated. In all patients, a blood sample was drawn simultaneously to determine the radioactivity in the blood.

Some of the bone marrow samples and blood samples were purified for the mononuclear cells (including the stem cells in the bone marrow samples). The samples (blood 8$48 \mathrm{mg}, 26 \pm 12.2 \mathrm{mg}$, mean $\pm \mathrm{SD}$; bone marrow $2-49 \mathrm{mg}$, $22 \pm 16.6 \mathrm{mg}$ ) were diluted with $5 \mathrm{ml}$ Dispase (Roche Diagnostics, Almere, The Netherlands) and mixed for 20 min to suspend the cells. Then the samples were diluted with $50 \mathrm{ml}$ phosphate-buffered saline (PBS) (pH 7.4) and centrifuged three times for $10 \mathrm{~min}$ at 2,500 rpm (about $60 \mathrm{~g}$ ) each time with washing with $50 \mathrm{ml}$ PBS after every step. The mononuclear cells, including the stem cells in the samples from the bone marrow, were isolated by FicollPaque gradient sedimentation (density $1.077 \mathrm{~g} / \mathrm{ml}$; GE Healthcare Europe, Diegem, The Netherlands). The weight of the cell pellets was then determined and the radioactivity was measured in a gamma counter.

\section{Bone marrow dosimetry}

For all patients the absorbed radiation dose to the bone marrow was calculated according to the MIRD scheme (Eq. 1). The radioactivity concentration in the red marrow was assumed to be equal to the radioactivity concentration measured in the blood $($ RMBLR $=1)$ [24, 31]. This was based on at least five blood samples per patient drawn between 0 and $168 \mathrm{~h}$ after injection. Assuming no specific uptake in the red marrow, a uniform distribution of the radioactivity, and that the red marrow clearance was the same as in blood, the self-dose to the red marrow was calculated. The distribution in the remainder of the body besides source organs and tumour uptake was assumed to be homogeneous. In addition, cross-radiation from the liver, spleen, kidneys, tumour as well as blood was taken into account. At three different time-points between 24 and $168 \mathrm{~h}$ after the administration of $\left[{ }^{177} \mathrm{Lu}-\mathrm{DOTA}^{0}, \mathrm{Tyr}^{3}\right]$ octreotate, scans of tumour deposits, liver, kidney and spleen were acquired using a dual head camera (Picker Prism 2000 XP, Philips, Eindhoven, The Netherlands) using the $208-\mathrm{keV}$ peak. The accumulated activity of the radiopharmaceutical in tumour and organs (liver, kidneys and spleen) was calculated from these scans. Attenuation correction was applied using the data from the pretreatment CT scan. It appeared that in our patient group there was a high degree of overprojection both for tumours and organs. As the S-factors of the liver, spleen and kidneys to the bone marrow were almost the same, the summation term in Eq. 1 was regarded as a single source organ.

Urine was collected up to $24 \mathrm{~h}$ after treatment and the radioactivity was measured in a gamma counter. From these data the accumulated activity in the remainder of the body (i.e. injected activity minus accumulated activity in source organs and tumours and minus excreted radioactivity) was calculated, taking into account the fact that the radioactivity was overestimated since the urine was collected for $24 \mathrm{~h}$ only. In two patients the radioactivity in the remainder of the body could not be determined. The quotient of $\widetilde{\mathrm{A}}$ and the injected activity IA yields the residence time $\tau=\widetilde{\mathrm{A}} / \mathrm{IA}$ (in hours) for the different organs. The thus calculated residence times were used as input in the OLINDA program (Vanderbilt University, Nashville, USA). 
Bone marrow dosimetry using SPECT scans

In addition, at three different time-points between 24 and $168 \mathrm{~h}$ after treatment, SPECT scans of the thorax were acquired (energy window $208 \mathrm{keV} \pm 10 \%$, matrix $128 \times 128$, 120 projections, $20 \mathrm{~s} /$ per projection).

Correlation with haematological response

Six weeks after the treatment, blood was drawn from 13 patients to determine haematological toxicity after the treatment. For one patient no blood results were available. All values were correlated with the decrease in platelet counts expressed as a percentage of the pretreatment value.

The results of the bone marrow (full bone marrow and nucleated cell fraction) radioactivity were corrected for physical decay in order to compare the interindividual values of the samples drawn at different time-points. Similarly, calculated absorbed dose to the bone marrow (self-dose and cross-dose) were correlated with the decrease in platelet counts.

\section{Statistics}

Pearson's correlation coefficient was calculated. A $p$ value $\leq 0.05$ was considered significant.

\section{Results}

The treatment with $\left[{ }^{177} \mathrm{Lu}-\mathrm{DOTA}{ }^{0}, \mathrm{Tyr}^{3}\right]$ octreotate was well tolerated by all patients and no serious adverse events occurred. The injected radioactivity ranged from 7.26 to $7.75 \mathrm{GBq}(7.47 \pm 0.10 \mathrm{GBq}$, mean $\pm \mathrm{SD})$. On the posttreatment scans all patients showed the expected distribution of the radiopharmaceutical with specific uptake in all known tumours. No patient had known or visible bone metastases.

The bone marrow aspirations were uneventful. One patient had a dry tap. The volume of bone marrow aspirated in the other patients ranged from 1 to $9.2 \mathrm{~g}(5.3 \pm 2.9 \mathrm{~g})$. Simultaneously a tube of blood of 2.8 to $7.3 \mathrm{~g}(5.8 \pm 1.3 \mathrm{~g})$ was drawn. Smears were made from the bone marrow aspirates. A differential count was performed to establish the numbers of immature (bone marrow) and mature nucleated cells. The fraction of immature cells ranged from $25 \%$ to $80 \%(51 \pm 15 \%)$ indicating that the purified aspirates consisted of a considerable amount of bone marrow and that the contamination with blood was moderate. A typical example of a purified bone marrow smear is shown in Fig. 1.

The radioactivity in the full bone marrow samples ranged from 850 to $4,473 \mathrm{~Bq} / \mathrm{ml}(2,216 \pm 899 \mathrm{~Bq} / \mathrm{ml})$. The radioactivity in the blood ranged from 1,077 to $6,451 \mathrm{~Bq} / \mathrm{ml}$ $(2,437 \pm 1,324 \mathrm{~Bq} / \mathrm{ml})$. Fitting the correlation line through

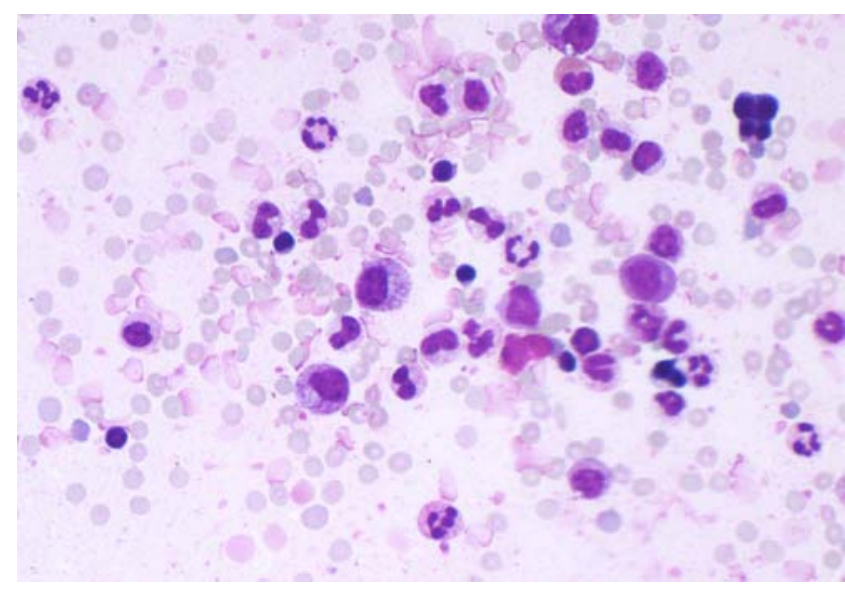

Fig. 1 Typical example of a smear of a bone marrow aspirate. It shows a mixture of immature nucleated cells originating from the bone marrow as well as red blood cells and mature nucleated cells originating from the peripheral blood

the origin $(0,0)$ as seems right from a theoretical point of view, showed a strong, significant, linear correlation between the radioactivity determined in the blood and in the bone marrow aspirate $(y=1.13 x, r=0.90, p<0.001$; Fig. 2). This results in a mean red marrow over blood ratio of 0.88 (value not significantly different from 1). Both qualitatively and quantitatively the results showed strong agreement over a whole range of activities at the three different time-points.

The self-absorbed doses to the bone marrow calculated from the accumulated activity in the blood (Eq. 2), calculated according to the MIRD scheme, and the changes in platelet counts after 6 weeks are presented in Table 1 . The contribution of the cross-dose to the red marrow dose was substantial in relation to the contribution of the selfdose (Table 1).

No or only very faint uptake in the bone marrow could be seen on the SPECT scans of the thorax. The bone marrow to background (ROI placed into the lungs) ratios were between 1 and 1.8. Therefore it was not possible to place a ROI reliably, and consequently no absorbed dose to the bone marrow was calculated from SPECT scans. An example of transaxial SPECT slices in one patient is shown in Fig. 3.

No correlation was found between the calculated absorbed doses to the bone marrow and the decrease in platelet counts, expressed as a percentage of the pretreatment value.

The correlation between the radioactivity in the full bone marrow aspirate and the decrease in platelet counts was poor ( $r=0.35, p=0.24$; Fig. 4). However, the correlation between the calculated absorbed doses and the decrease in platelet counts was even less significant.

The ratios of the radioactivity in the isolated mononuclear cell fraction of the bone marrow to the isolated mononuclear 


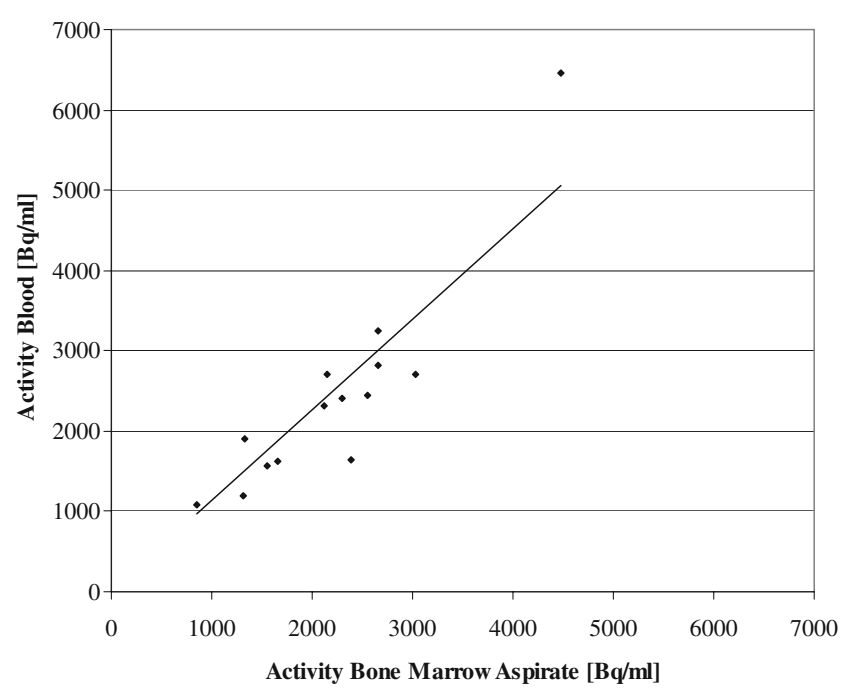

Fig. 2 Correlation between the activities measured in the bone marrow aspirate and the activities measured in the blood at the same time-points. The straight line is the linear regression line: $r=0.914$, $p<0.001$. The slope of the regression line is 1.35 indicating that the absolute values of the measured activities are also comparable cell fraction of the blood obtained on the same day were highly variable. We found ratios ranging from 0.29 to 536 (59.3 \pm 143.6$)$. In three patients a higher radioactivity was found in the mononuclear cells isolated from the blood whereas 11 patients showed a higher radioactivity in the mononuclear cells isolated from the bone marrow.

In 11 patients the radioactivity in the isolated mononuclear cell fraction, microscopically containing a mixture of immature and mature cells, could be weight-corrected. In the other three patients, the isolated fraction could not be weighted reliably because of the small number of isolated cells. Weight-corrected ratios of the radioactivity in the isolated cell fraction of the bone marrow to the isolated cell fraction of the blood ranged from 0.54 to 133.41 (19.4 \pm 38.6$)$. Only one patient showed a higher radioactivity in the isolated cell fraction per gram of blood. The correlation coefficient between the radioactivity per gram in the nucleated cell fraction of the bone marrow aspirate and the decrease in platelet count after treatment was $r=0.51$ ( $p=0.13$; Fig. 5). From our study this was the best relationship that could be obtained.

Table 1 Doses to the bone marrow after injection of 3,700 MBq $\left[{ }^{177} \mathrm{Lu}-\mathrm{DOTA}{ }^{0}\right.$, Tyr $\left.{ }^{3}\right]$ octreotate, and decrease in platelet counts 6 weeks after treatment

\begin{tabular}{|c|c|c|c|c|c|}
\hline \multirow[t]{2}{*}{ Patient } & \multirow{2}{*}{$\begin{array}{l}\text { Total absorbed dose to } \\
\text { the red marrow }(\mathrm{mGy})^{\mathrm{a}}\end{array}$} & \multirow{2}{*}{$\begin{array}{l}\text { Absorbed self- } \\
\text { dose }(m G y)^{b}\end{array}$} & \multicolumn{2}{|l|}{ Cross-dose $(\mathrm{mGy})^{\mathrm{c}}$} & \multirow{2}{*}{$\begin{array}{l}\text { Decrease in platelet count } \\
6 \text { weeks after treatment }(\%)\end{array}$} \\
\hline & & & $\begin{array}{l}\text { From the remainder } \\
\text { of the body }\end{array}$ & $\begin{array}{l}\text { From } \\
\text { organs }\end{array}$ & \\
\hline 1 & 40 & $16(41 \%)$ & $12(31 \%)$ & $11(29 \%)$ & 40 \\
\hline 2 & 82 & $37(46 \%)$ & $26(32 \%)$ & $19(23 \%)$ & 8 \\
\hline 3 & 212 & $49(23 \%)$ & $152(72 \%)$ & $11(5 \%)$ & 40 \\
\hline 4 & 108 & $17(16 \%)$ & $83(77 \%)$ & $8(8 \%)$ & 16 \\
\hline 5 & 64 & $23(36 \%)$ & $26(41 \%)$ & $15(23 \%)$ & 4 \\
\hline 6 & 129 & $37(28 \%)$ & $78(61 \%)$ & $14(11 \%)$ & - \\
\hline 7 & 73 & $28(39 \%)$ & $0(0 \%)$ & $45(61 \%)$ & -9 \\
\hline 8 & 74 & $39(53 \%)$ & $28(39 \%)$ & $6(9 \%)$ & -9 \\
\hline 9 & & 29 & - & - & 47 \\
\hline 10 & 466 & $26(5 \%)$ & $429(92 \%)$ & $11(2 \%)$ & 8 \\
\hline 11 & 147 & $56(38 \%)$ & $84(58 \%)$ & $7(5 \%)$ & 27 \\
\hline 12 & 78 & $23(30 \%)$ & $39(50 \%)$ & $16(20 \%)$ & 32 \\
\hline 13 & 86 & $29(34 \%)$ & $39(45 \%)$ & $18(21 \%)$ & 28 \\
\hline 14 & 83 & $24(29 \%)$ & $48(57 \%)$ & $12(14 \%)$ & 21 \\
\hline Median & 83 & $29(34 \%)$ & $39(50 \%)$ & $12(14 \%)$ & 21 \\
\hline Mean & 126 & $31(32 \%)$ & $80(50 \%)$ & $15(18 \%)$ & 19 \\
\hline $\mathrm{SD}$ & 111 & $12(12 \%)$ & $112(24 \%)$ & $10(16 \%)$ & 18 \\
\hline
\end{tabular}

${ }^{a}$ Taking into account the absorbed self-dose, the cross-dose from the remainder of the body and the absorbed dose of organs and tumour.

${ }^{\mathrm{b}}$ From the red marrow to the red marrow.

${ }^{\mathrm{c}}$ To the red marrow.

${ }^{\mathrm{d}}$ Liver, spleen, kidneys and tumour. 
ant.

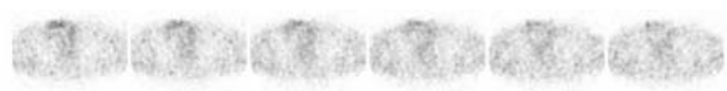

right

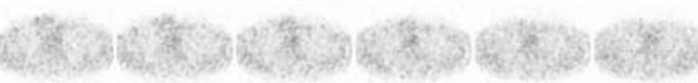

left

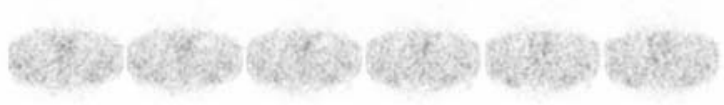

post.

Fig. 3 Typical example of transverse SPECT slices through the thorax obtained $24 \mathrm{~h}$ after treatment with $7.4 \mathrm{GBq}\left[{ }^{177} \mathrm{Lu}-\mathrm{DOTA}{ }^{0}, \mathrm{Tyr}^{3}\right]$ octreotate. Note that there is no clear uptake over the spine area

\section{Discussion}

Accurate bone marrow dosimetry is mandatory for safe PRRT. All methods available have certain disadvantages: aspiration of bone marrow is costly and time consuming, and it is an invasive procedure associated with a high level of discomfort for the patients. Calculating the dose to the red marrow from the accumulated activity concentration in the blood deals with the assumption that no specific binding of the radiolabelled somatostatin analogue occurs in the bone marrow. Also, it neglects the contribution of other radiation sources in the body, such as organs and tumours, thereby underestimating the true radiation absorbed dose to the bone marrow. Alternatively, the determination of the radioactivity in the remainder of the body (needed for the MIRD scheme) depends on accuracy in urine collection, which is subject to errors. Apart from these considerations, it should be realized that at present none of the diverse models to estimate the dose to the bone marrow has been actually confirmed with toxicity data, be it the incidence of thrombocytopenia or the occurrence of MDS. Apart from the risk of patients developing MDS, the essential issue of

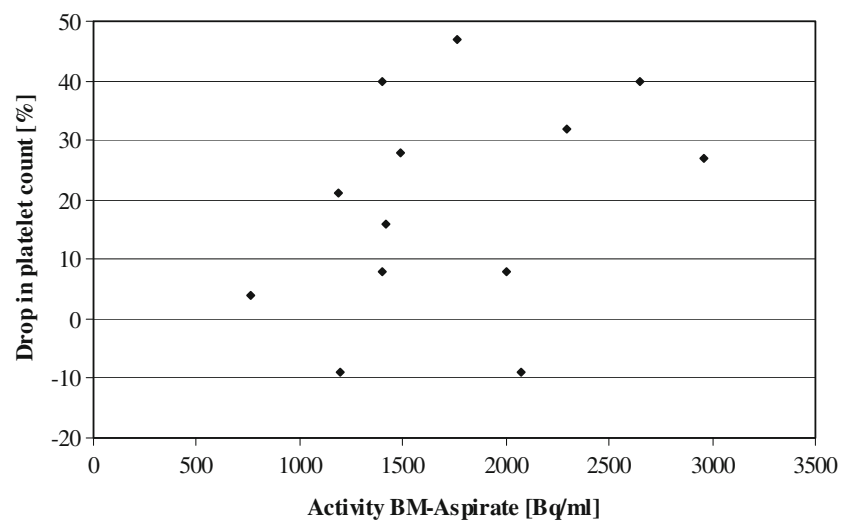

Fig. 4 Plot of the radioactivity in the bone marrow aspirate versus the percentage decrease in platelet count. The activities measured in the bone marrow were decay-corrected to the theoretical value on day 8 . Linear regression coefficient: $r=0.35(p=0.24)$ bone marrow dosimetry is to predict, or better to avoid, severe haematological toxicity caused by PRRT.

The dose to the red marrow, however, is only one of several factors influencing the haematological toxicity after PRRT. A high interpatient variability in the haematological response after PRRT has been found and also previous therapies can highly influence the results [2, 29]. Using different radiopharmaceuticals, some investigators have found a correlation between haematological toxicity and injected dose of radioactivity [32,33], whereas others have not found such a correlation [34]. It is possible that additional factors such as age and sex of the patients might influence the haematological toxicity as well, although in a trial with radiolabelled antibodies there was only a minor influence of these factors [35]. As early as the year 2000, Blumenthal et al reported that plasma levels of FLT3-L help to predict haematological toxicity after radioimmunotherapy [36]. In 2003 this was confirmed by Siegel et al. for radioimmunotherapy with iodinated anti-CEA antibodies [37]. However, no studies taking this into account have been published for PRRT. Nevertheless, the importance of introducing biological parameters into treatment planning is indisputable [38].

We found a high correlation between the radioactivity concentration in the blood and in the bone marrow aspirate during PRRT with $\left[{ }^{177} \mathrm{Lu}-\mathrm{DOTA}{ }^{0}, \mathrm{Tyr}^{3}\right]$ octreotate. The most probable explanation for the high congruence between the radioactivity measured in the blood and in the bone marrow aspirate is that the amount of stem cells in a bone marrow aspirate is low and that most of the aspirate consists of blood. On the other hand, the high volume of blood in the bone marrow aspirate reflects the fact that the blood contributes most of the self-dose to the bone marrow. Taking into consideration that the path lengths of the common therapeutic radionuclides are in the millimetre

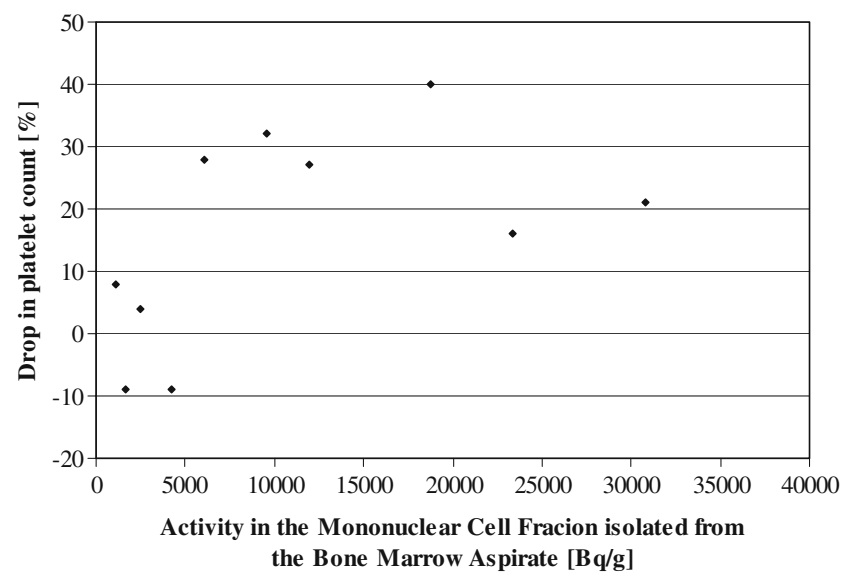

Fig. 5 Correlation between the radioactivity per gram measured in the isolated mononuclear cell fraction of the bone marrow aspirate with the decrease in platelet count after treatment. Linear regression coefficient: $r=0.51(p=0.13)$ 
range and the structure of the bone marrow it is evident that the radiation from the blood will reach all bone marrow structures.

Promising results for predicting the haematological response were obtained by using an ROI surrounding a section of the thoracic spine for determining the absorbed dose to the red marrow. In these studies the absorbed dose was calculated with the positron emitter ${ }^{86} \mathrm{Y}$ before treatment with ${ }^{90}$ Y-DOTATOC $[29,39] .{ }^{86} \mathrm{Y}$ can be regarded as the ideal surrogate for ${ }^{90} \mathrm{Y}$ and offers a high resolution when using a PET scanner. However, imaging with ${ }^{86} \mathrm{Y}$ is currently only available in specialized centres because besides the need for a cyclotron to produce ${ }^{86} \mathrm{Y}$, the reconstruction of the PET data requires sophisticated correction algorithms. There is certain evidence in literature that imaging with ${ }^{86} \mathrm{Y}$ might overestimate doses, particularly if the bone marrow is close to dense tissue such as the spine [40, 41]. Besides, dosimetry using ${ }^{86} \mathrm{Y}$ will be a gold standard only for treatments with ${ }^{90} \mathrm{Y}$ because in PRRT the radionuclide used influences the receptor affinity and consequently the biodistribution of the compound [42]. Moreover, the relatively short physical half-life of ${ }^{86} \mathrm{Y}$ $(14.7 \mathrm{~h})$ does not allow the radioactivity to be followed over several days which is important for planning of the treatment with ${ }^{90} \mathrm{Y}$. However, these results have not yet been confirmed by another group.

To date one group has found interesting results when calculating the absorbed radiation dose to the bone marrow from scans for radiolabelled antibodies using an integrated SPECT/CT camera [30]. The results have also not been confirmed by other groups, and no results using this method with radiopeptides have been published so far. Remarkably no bone marrow dosimetry was feasible on SPECT scans in our setting because no or only very faintly visible uptake in the bone marrow was present on the scans (Fig. 3).

Our results indicate the radioactivity in the blood is a very accurate surrogate for the radioactivity present in the bone marrow. However, we also showed that the radioactivity in the remainder of the body as well as the radioactivity in source organs and tumours significantly contributes to the absorbed radiation dose to the bone marrow. With that, our tacit hope that the cumbersome collection of 24-h urine and time-consuming calculations of cross-radiation from tumours and organs could be circumvented, and that bone marrow absorbed doses could be derived from blood radioactivity levels only, evaporated. Also, the bone marrow absorbed dose after treatment with $\left[{ }^{177} \mathrm{Lu}-D O T A^{0}, \mathrm{Tyr}^{3}\right]$ octreotate showed considerable variation between patients. This is in contrast with the findings of a previous pilot study in six patients that showed the biodistribution of the radiopharmaceutical [43]. These patients, however, all had a very limited tumour load. The present series of patients more truly represented the practice of PRRT in our institution, where the tumour load in patients is highly variable, and so the contribution of the cross-dose to the bone marrow is variable as well. This finding implies that for individual dose optimization, individual calculation of the bone marrow absorbed dose is necessary. The results shown in Table 1 indicate that the remainder of the body as well as organs and tumours significantly contribute to the absorbed dose to the bone marrow. Since the contribution to this dose from the remainder of the body, organs and tumours from the $\beta$-emission of ${ }^{177} \mathrm{Lu}$ is virtually negligible, it appears that the $\gamma$-radiation from tumours, organs and the remainder of the body contributes to the absorbed bone marrow dose.

We determined the degree of correlation between the absorbed dose to the red marrow and the decrease in platelet count after 6 weeks. We focused on platelets only since platelets show the most pronounced reaction after PRRT. The degree of correlation between the calculated absorbed doses to the red marrow and the decrease in platelet count was disappointing. A number of reasons may have accounted for this. The absorbed doses were compared with only one posttreatment platelet count. This platelet count, 6 weeks after treatment, may not have reflected the nadir in each patient. Another explanation could have been the relatively small number of patients studied. Moreover, probably the most important reason could have been that the response of an individual patient to PRRT is not only related to the radiation absorbed dose in the bone marrow but also to the pretreatment status of the bone marrow. Especially previous, potentially haemocytotoxic treatments can influence the response after the treatment.

No conclusions can be drawn concerning the relationship between the calculated radiation absorbed dose in the bone marrow and the risk of developing MDS. However, developing MDS is probably also related to the pretreatment status of the bone marrow and previous treatments.

Bone marrow dosimetry is a difficult topic. Beside all factors mentioned above that may influence the dosimetry of an individual patient, many other considerations have to be faced. The bone marrow is not a solid organ and simply the determination of the mass is virtually impossible. As for all internal radiotherapy treatments, the dose rate in PRRT is low. Most values that deal with the maximum tolerated dose of healthy organs are derived from external beam radiation with a much higher dose rate. The influence of such physical properties is not well understood and may highly influence the results of internal dosimetry as much as the biological response.

In conclusion, our results show that: (1) after PRRT with $\left[{ }^{177} \mathrm{Lu}-\mathrm{DOTA}^{0}, \mathrm{Tyr}^{3}\right]$ octreotate, the radioactivity concentration in the bone marrow is identical to that in the blood; (2) there is no significant binding of the radiopharmaceutical to bone marrow precursor stem cells; (3) the contribution of 
the cross-dose from source organs and tumours to the bone marrow dose is significant; (4) there is considerable variation in bone marrow absorbed dose between patients. These findings imply that for individual dose optimization, individual calculation of the bone marrow absorbed dose is necessary.

Acknowledgments F. Forrer received a personal research grant from the Swiss National Science Foundation and the Novartis Foundation. The authors wish to thank all the supporting personnel of the Department of Nuclear Medicine and the Department of Internal Medicine for their help and effort. We also wish to thank Dr. Stephan Walrand (Nuclear Medicine Center, Catholic University of Louvain, Brussels, Belgium) for highly valuable discussions.

Conflict of interest None.

Open Access This article is distributed under the terms of the Creative Commons Attribution Noncommercial License which permits any noncommercial use, distribution, and reproduction in any medium, provided the original author(s) and source are credited.

\section{References}

1. Waldherr C, Pless M, Maecke H, Schumacher T, Crazzolara A, Nitzsche EU, et al. Tumor response and clinical benefit in neuroendocrine tumors after 7.4 GBq 90Y-DOTATOC. J Nucl Med 2002;43:610-16.

2. Kwekkeboom DJ, Bakker WH, Kam BL, Teunissen JJ, Kooij PP, de Herder WW, et al. Treatment of patients with gastro-enteropancreatic (GEP) tumours with the novel radiolabelled somatostatin analogue [177Lu-DOTA(0),Tyr(3)]octreotate. Eur J Nucl Med 2003;30:417-22.

3. Otte A, Herrmann R, Heppeler A, Behe M, Jermann E, Powell P, et al. Yttrium-90 DOTATOC: first clinical results. Eur J Nucl Med 1999;26:1439-47.

4. Forrer F, Uusijarvi H, Storch D, Maecke HR, Mueller-Brand J. Treatment with 177Lu-DOTATOC of patients with relapse of neuroendocrine tumors after treatment with 90Y-DOTATOC. J Nucl Med 2005;46:1310-16.

5. Kwekkeboom DJ, Mueller-Brand J, Paganelli G, Anthony LB, Pauwels S, Kvols LK, et al. Overview of results of peptide receptor radionuclide therapy with 3 radiolabeled somatostatin analogs. J Nucl Med 2005;46(Suppl 1):62S-66S.

6. Kwekkeboom DJ, Teunissen JJ, Bakker WH, Kooij PP, de Herder WW, Feelders RA, et al. Radiolabeled somatostatin analog [177Lu-DOTA $(0), \operatorname{Tyr}(3)]$ octreotate in patients with endocrine gastroenteropancreatic tumors. J Clin Oncol 2005;23:2754-62.

7. Paganelli G, Bodei L, Handkiewicz Junak D, Rocca P, Papi S, Lopera Sierra M, et al. 90Y-DOTA-D-Phe(1)-Try(3)-octreotide in therapy of neuroendocrine malignancies. Biopolymers 2002;66:393-8.

8. Moll S, Nickeleit V, Mueller-Brand J, Brunner FP, Maecke HR, Mihatsch MJ. A new cause of renal thrombotic microangiopathy: yttrium 90-DOTATOC internal radiotherapy. Am J Kidney Dis 2001;37:847-51.

9. Valkema R, Pauwels SA, Kvols LK, Kwekkeboom DJ, Jamar F, de Jong $\mathrm{M}$, et al. Long-term follow-up of renal function after peptide receptor radiation therapy with $(90)$ Y-DOTA(0), Tyr(3)octreotide and (177)Lu-DOTA(0), Tyr(3)-octreotate. J Nucl Med 2005;46(Suppl 1):83S-91S.
10. Jamar F, Barone R, Mathieu I, Walrand S, Labar D, Carlier P, et al. 86Y-DOTA0)-D-Phe1-Tyr3-octreotide (SMT487) - a phase 1 clinical study: pharmacokinetics, biodistribution and renal protective effect of different regimens of amino acid co-infusion. Eur J Nucl Med Mol Imaging 2003;30:510-8.

11. Barone R, Borson-Chazot F, Valkema R, Walrand S, Chauvin F, Gogou L, et al. Patient-specific dosimetry in predicting renal toxicity with (90)Y-DOTATOC: relevance of kidney volume and dose rate in finding a dose-effect relationship. J Nucl Med 2005;46(Suppl 1):99S-106S.

12. Konijnenberg MW, Bijster M, Krenning EP, De Jong M. A stylized computational model of the rat for organ dosimetry in support of preclinical evaluations of peptide receptor radionuclide therapy with (90)Y, (111)In, or (177)Lu. J Nucl Med 2004;45:1260-9.

13. Esser JP, Krenning EP, Teunissen JJ, Kooij PP, van Gameren AL, Bakker WH, et al. Comparison of [(177)Lu-DOTA(0),Tyr(3)]octreotate and [(177)Lu-DOTA $(0), \operatorname{Tyr}(3)]$ octreotide: which peptide is preferable for PRRT? Eur J Nucl Med Mol Imaging 2006;33:1346-51.

14. Valkema R, De Jong M, Bakker WH, Breeman WA, Kooij PP, Lugtenburg PJ, et al. Phase I study of peptide receptor radionuclide therapy with [In-DTPA] octreotide: the Rotterdam experience. Semin Nucl Med 2002;32:110-22.

15. Kwekkeboom DJ, Bakker WH, Teunissen JJM, Kooij PP, Krenning EP. Treatment with Lu-177-DOTA-Tyr3-octreotate in patients with neuroendocrine tumors: interim results (abstract). Eur J Nucl Med Mol Imaging 2003;30(Suppl 2):S231.

16. Kwekkeboom DJ, de Herder WW, Kam BL, van Eijck CH, van Essen M, Kooij PP, et al. Treatment with the radiolabelled

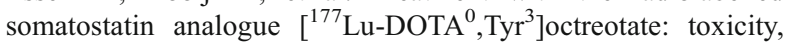
efficacy, and survival. J Clin Oncol 2008;26:2124-30.

17. ICRP. Publication 41: nonstochastic effects of ionizing radiation. Pergamon Press, Oxford, 1984.

18. Coleman CN, Blakely WF, Fike JR, MacVittie TJ, Metting NF, Mitchell JB, et al. Molecular and cellular biology of moderatedose (1-10 Gy) radiation and potential mechanisms of radiation protection: report of a workshop at Bethesda, Maryland, December 17-18, 2001. Radiat Res 2003;159:812-34.

19. Benua RS, Cicale NR, Sonenberg M, Rawson RW. The relation of radioiodine dosimetry to results and complications in the treatment of metastatic thyroid cancer. AJR Am J Roentgenol 1962;87:171-82.

20. Dorn R, Kopp J, Vogt H, Heidenreich P, Carroll RG, Gulec SA. Dosimetry-guided radioactive iodine treatment in patients with metastatic differentiated thyroid cancer: largest safe dose using a risk-adapted approach. J Nucl Med 2003;44:451-6.

21. Siegel JA, Wessels BW, Watson EE, Stabin MG, Vriesendorp $\mathrm{HM}$, Bradley EW, et al. Bone marrow dosimetry and toxicity for radioimmunotherapy. Antibody Immunoconjug Radiopharm 1990;3:213-33.

22. Wessels BW, Bolch WE, Bouchet LG, Breitz HB, DeNardo GL, Meredith RF, et al. Bone marrow dosimetry using blood-based models for radiolabeled antibody therapy: a multiinstitutional comparison. J Nucl Med 2004;45:1725-33.

23. Cremonesi M, Ferrari M, Zoboli S, Chinol M, Stabin MG, Orsi F, et al. Biokinetics and dosimetry in patients administered with (111)In-DOTA-Tyr(3)-octreotide: implications for internal radiotherapy with (90)Y-DOTATOC. Eur J Nucl Med 1999;26:87786.

24. Sgouros G. Bone marrow dosimetry for radioimmunotherapy: theoretical considerations. J Nucl Med 1993;34:689-94.

25. Shen S, DeNardo SJ, Richman CM, Yuan A, Siantar $\mathrm{CH}$, O'Donnell RT, et al. Planning time for peripheral blood stem cell infusion after high-dose targeted radionuclide therapy using dosimetry. J Nucl Med 2005;46:1034-41.

26. Reubi JC, Waser B, Schaer JC, Laissue JA. Somatostatin receptor sst1-sst5 expression in normal and neoplastic human tissues using 
receptor autoradiography with subtype-selective ligands. Eur J Nucl Med 2001;28:836-46.

27. Lichtenauer-Kaligis EG, Dalm VA, Oomen SP, Mooij DM, van Hagen PM, Lamberts SW, et al. Differential expression of somatostatin receptor subtypes in human peripheral blood mononuclear cell subsets. Eur J Endocrinol 2004;150:565-77.

28. Oomen SP, van Hennik PB, Antonissen C, Lichtenauer-Kaligis EG, Hofland LJ, Lamberts SW, et al. Somatostatin is a selective chemoattractant for primitive (CD34(+)) hematopoietic progenitor cells. Exp Hematol 2002;30:116-25.

29. Pauwels S, Barone R, Walrand S, Borson-Chazot F, Valkema R, Kvols LK, et al. Practical dosimetry of peptide receptor radionuclide therapy with (90)Y-labeled somatostatin analogs. J Nucl Med 2005;46(Suppl 1):92S-98S.

30. Boucek JA, Turner JH. Validation of prospective whole-body bone marrow dosimetry by SPECT/CT multimodality imaging in 131I-anti-CD20 rituximab radioimmunotherapy of non-Hodgkin's lymphoma. Eur J Nucl Med Mol Imaging 2005;32:458-69.

31. Stabin MG, Siegel JA, Sparks RB, Eckerman KF, Breitz HB. Contribution to red marrow absorbed dose from total body activity: a correction to the MIRD method. J Nucl Med 2001;42:492-8.

32. Matthay KK, Panina C, Huberty J, Price D, Glidden DV, Tang HR, et al. Correlation of tumor and whole-body dosimetry with tumor response and toxicity in refractory neuroblastoma treated with (131)I-MIBG. J Nucl Med 2001;42:1713-21.

33. Lim SM, DeNardo GL, DeNardo DA, Shen S, Yuan A, O'Donnell RT, et al. Prediction of myelotoxicity using radiation doses to marrow from body, blood and marrow sources. J Nucl Med 1997;38:1374-78.

34. Vallabhajosula S, Goldsmith SJ, Hamacher KA, Kostakoglu L, Konishi S, Milowski MI, et al. Prediction of myelotoxicity based on bone marrow radiation-absorbed dose: radioimmunotherapy studies using 90Y- and 177Lu-labeled J591 antibodies specific for prostate-specific membrane antigen. J Nucl Med $2005 ; 46: 850-58$.
35. Juweid ME, Zhang CH, Blumenthal RD, Hajjar G, Sharkey RM, Goldenberg DM. Prediction of hematologic toxicity after radioimmunotherapy with (131)I-labeled anticarcinoembryonic antigen monoclonal antibodies. J Nucl Med 1999;40:1609-616.

36. Blumenthal RD, Lew W, Juweid M, Alisauskas R, Ying Z, Goldenberg DM. Plasma FLT3-L levels predict bone marrow recovery from myelosuppressive therapy. Cancer 2000;88:333-43.

37. Siegel JA, Yeldell D, Goldenberg DM, Stabin MG, Sparks RB, Sharkey RM, et al. Red marrow radiation dose adjustment using plasma FLT3-L cytokine levels: improved correlations between hematologic toxicity and bone marrow dose for radioimmunotherapy patients. J Nucl Med 2003;44(1):67-76.

38. Sgouros G. Dosimetry of internal emitters. J Nucl Med 2005;46 (Suppl 1):18S-27S.

39. Walrand S, Barone R, Jamar F, De Camps J, Krenning EP, Valkema R, et al. Red marrow 90Y-OctreoTher dosimetry estimated using 86Y-OctreoTher PET and biological correlates (abstract). Eur J Nucl Med Mol Imaging 2002;29(Suppl 1):301S.

40. Pentlow KS, Finn RD, Larson SM, Erdi YE, Beattie BJ, Humm JL. Quantitative imaging of yttrium- 86 with PET. The occurrence and correction of anomalous apparent activity in high density regions. Clin Positron Imaging. 2000;3:85-90.

41. Buchholz HG, Herzog H, Forster GJ, Reber H, Nickel O, Rosch F, et al. PET imaging with yttrium-86: comparison of phantom measurements acquired with different PET scanners before and after applying background subtraction. Eur J Nucl Med Mol Imaging 2003;30:716-20.

42. Reubi JC, Schar JC, Waser B, Wenger S, Heppeler A, Schmitt JS, et al. Affinity profiles for human somatostatin receptor subtypes SST1-SST5 of somatostatin radiotracers selected for scintigraphic and radiotherapeutic use. Eur J Nucl Med 2000;27:273-82.

43. Kwekkeboom DJ, Bakker WH, Kooij PP, Konijnenberg MW, Srinivasan A, Erion JL, et al. [177Lu-DOTA(0)Tyr(3)]octreotate: comparison with [111In-DTPA(0)]octreotide in patients. Eur $\mathrm{J}$ Nucl Med 2001;28:1319-25. 